\title{
FUNCTIONS OF WELL-BOUNDED OPERATORS
}

\author{
HAROLD E. BENZINGER ${ }^{1}$
}

\begin{abstract}
It is shown that if $A$ is a well-bounded operator of type (B), and if $f$ is of bounded variation and piecewise monotone, then $f(A)$ is also well bounded of type (B).
\end{abstract}

1. Introduction. Let $C$ be a simple, nonclosed rectifiable arc in the complex plane. For a complex valued function $f$ defined on $C$, let

$$
\|f\|_{C}=\sup (|f|, C)+\operatorname{var}(f, C),
$$

where $\sup (|f|, C)$ is the supremum on $C$ of $|f|$, and $\operatorname{var}(f, C)$ is the total variation of $f$ on $C$. Let $X$ denote a complex Banach space and let $A$ denote a bounded linear operator on $X$.

1.1. Definition. The operator $A$ is well bounded if there exist an arc $C$ and a constant $K>0$ such that for all polynomials $p,\|p(A)\| \leqslant K\|p\|_{C}$.

Well-bounded operators are important in the spectral theory of linear operators with spectral expansions which are only conditionally convergent, e.g. Fourier expansions in $L^{p}$ spaces, $p \neq 2$. See [1] for applications to groups and semigroups of linear operators, and to ordinary differential operators. $\$ 2$ of [1] contains a summary of the theory of well-bounded operators. A systematic development is found in [2, Part 5]. The value of the theory arises from the existence of a functional calculus: a homomorphism from a Banach algebra of functions on $C$ into the algebra of bounded linear operators on $X$. Furthermore, there is a modified Riemann-Stieltjes integral on $C$ which gives a representation of the homomorphism. See below, and also [1, Propositions 2.1, 2.3]. The functional calculus always exists on the algebra of absolutely continuous functions on $C$, but for the special class of well-bounded operators of type (B) (see below, and also [2, p. 315]), and thus always in the case that $X$ is reflexive, the functional calculus can be extended to $\operatorname{BV}(C)$, the algebra of functions of bounded variation on $C$, with norm $\|f\|_{C}$.

In this paper we show that for well-bounded operators of type (B), there is a substantial subset of functions $f$ in $\operatorname{BV}(C)$ such that $f(A)$ is also well bounded.

Since the $\operatorname{arc} C$ is the image of a finite, closed real interval under the arc-length parameterization, there is no loss of generality in assuming that $C$ is in fact a real interval (see [1, Proposition 2.8]).

Received by the editors September 19, 1983.

1980 Mathematics Subject Classification. Primary 47A60.

${ }^{1}$ Part of this work was done while the author was on sabbatical leave at the University of Duisburg and the Technical University of Aachen, West Germany. 
1.2. Definition. A spectral family on the interval $J=[a, b]$ is a projection-valued function $E: J \rightarrow \mathscr{B}(X)$ (the space of bounded linear operators on $X$ ) satisfying:

(i) $\sup \{\|E(\lambda)\|: \lambda \in J\}=K<\infty$;

(ii) $E(\lambda) E(\mu)=E(\mu) E(\lambda)=E(\min (\lambda, \mu))$ for $\lambda, \mu \in J$;

(iii) $E(\lambda)$ is right continuous on $J$ in the strong operator topology;

(iv) $E(\lambda)$ has a left-hand limit in the strong operator topology at each point of $J$;

(v) $E(b)=I$ (the identity operator on $X$ ).

For $f \in \mathrm{BV}(J)$, let $\int_{J}^{r} f(\lambda) d E(\lambda)$ denote the strong limit of Riemann-Stieltjes sums such that the intermediate point in each interval is the right endpoint. For the proof that this limit exists, see [2, Chapter 17]. Let

$$
\int_{J}^{\oplus} f(\lambda) d E(\lambda)=f(a) E(a)+\int_{J}^{r} f(\lambda) d E(\lambda) .
$$

1.4. TheOREM [2, ChAPTER 17]. The bounded linear operator $A$ is a well-bounded operator of type (B) on $J$ if and only if there exists a spectral family $E(\lambda)$ on $J$ such that

$$
A=\int_{J}^{\oplus} \lambda d E(\lambda)
$$

For every $f \in \mathrm{BV}(J)$,

$$
f(A)=\int_{J}^{\oplus} f(\lambda) d E(\lambda)
$$

defines an algebra homomorphism of $\mathrm{BV}(J)$ into $\mathscr{B}(X)$, such that

$$
\|f(A)\| \leqslant K\|f\|_{J} .
$$

1.8. Definition. A real-valued function $f$ on $J$ is piecewise monotone if $J$ is the union of finitely many intervals $J_{i}=\left[a_{i-1}, a_{i}\right], i=1, \ldots, m$, with $a_{0}=a, a_{m}=b$, such that $f$ is monotone on each $J_{i}$.

We shall prove the following theorem.

1.9. ThEOREM. If $f$ in $\mathrm{BV}(J)$ is piecewise monotone, and if $A$ is a well-bounded operator of type (B) on $J$, then $f(A)$ is a well-bounded operator of type (B) (on any compact interval containing $f(J))$.

1.10. REMARK. For the case $f$ is strictly monotone and continuous, see [1, Lemma 4.3].

The author wishes to acknowledge a helpful conversation with Professor Earl Berkson.

2. The case that $f$ is monotone. In this section we prove Theorem 1.9 in the case that $f$ is monotone on all of $J$. Let $A$ be a well-bounded operator of type (B) on $J$ with spectral family $\{E(\lambda): \lambda \in J\}$. Assume $f$ is bounded and monotone nondecreasing on $J$, with $\alpha=f(a), \beta=f(b), H=[\alpha, \beta]$. For $\mu$ in $H$, define

$$
S(\mu)=\{\lambda \in J: F(\lambda) \leqslant \mu\}, \quad \lambda(\mu)=\sup \{\lambda: \lambda \in S(\mu)\} .
$$


Note that

$$
S(\mu)= \begin{cases}{[a, \lambda(\mu)]} & \text { if } f \text { is left continuous at } \lambda(\mu), \\ {[a, \lambda(\mu))} & \text { otherwise. }\end{cases}
$$

We define a family of projections $F(\mu)$ on $H$ by

$$
F(\mu)= \begin{cases}E(\lambda(\mu)) & \text { if } f \text { is left continuous at } \lambda(\mu), \\ E\left(\lambda^{-}(\mu)\right) & \text { otherwise. }\end{cases}
$$

$\left(E\left(\lambda_{0}^{-}\right) x=\lim _{\lambda \uparrow \lambda_{0}} E(\lambda) x\right.$, which exists for all $x$ in $X$ and all $\lambda_{0}$ in $J$ by property (iv) of a spectral family.)

Let $\left\{\nu_{k}: k=1,2, \ldots\right\}$ denote the at most countable set in $J$ where $f$ fails to be left continuous, and define

$$
\begin{gathered}
\gamma_{k}=\lim _{\lambda \uparrow \nu_{k}} f(\lambda), \quad \rho_{k}=f\left(\nu_{k}\right)-\gamma_{k}>0, \\
h(\lambda)=\left\{\begin{array}{ll}
0, & \lambda \neq \nu_{k}, \\
\rho_{k}, & \lambda=\nu_{k}
\end{array} \quad g=f-h .\right.
\end{gathered}
$$

Then $g$ is left continuous on $J$. (If $\nu_{k}$ is a cluster point of the set of left discontinuities, we use the fact that $\sum \rho_{k}<\infty$.)

2.6. LEMMA. $\{F(\mu): \mu \in H\}$ is a spectral family on $H$.

Proof. $F(\mu)$ clearly satisfies properties (i), (ii), (v) of Definition 1.2. Property (iv) holds for $F$ since $\lambda(\mu)$ is a monotone nondecreasing function of $\mu$, and since (iv) holds for $E$. To establish the right continuity of $F$, let $\mu_{0} \in H, x \in X$. Let $\lambda_{0}=\lim _{\mu \downarrow \mu_{0}} \lambda(\mu)$. Then $\lambda_{0}=\lambda\left(\mu_{0}\right)$, since otherwise there exists $\lambda, \lambda\left(\mu_{0}\right)<\lambda<\lambda_{0}$, such that $f(\lambda)>\mu_{0}$ but $f(\lambda) \leqslant \mu$ for all $\mu>\mu_{0}$. Suppose first that $f$ is left continuous at $\lambda_{0}=\lambda\left(\mu_{0}\right)$. Then for $\mu>\mu_{0}$,

$$
F(\mu) x-F\left(\mu_{0}\right) x= \begin{cases}E(\lambda(\mu)) x-E\left(\lambda_{0}\right) x & \text { if } f \text { is left continuous at } \lambda(\mu), \\ E\left(\lambda^{-}(\mu)\right) x-E\left(\lambda_{0}\right) x & \text { otherwise. }\end{cases}
$$

In each case, the right continuity of $E(\lambda)$ guarantees that this vector can be made arbitrarily small by selecting $\mu>\mu_{0}$ sufficiently close to $\mu_{0}$. Now consider the case that $f$ is not left continuous at $\lambda_{0}$. Then $f\left(\lambda_{0}\right)>\lim _{\lambda \uparrow \lambda_{0}} f(\lambda)=\gamma_{0}$, so for $\gamma_{0} \leqslant \mu<$ $f\left(\lambda_{0}\right), F(\mu)$ is constant and therefore right continuous.

Let $B$ denote the well-bounded operator of type (B) on $H$ given by

$$
B=\int_{H}^{\oplus} \mu d F(\mu)
$$

We prove that $f(A)$ is well bounded by showing

2.8. Lemma. $f(A)=B$.

Proof. First we note that since $\sum \rho_{k}<\infty$ and $\|E(\lambda)\| \leqslant K$, we have

$$
h(A)=\sum_{1}^{\infty} \rho_{k}\left[E\left(\nu_{k}\right)-E\left(\nu_{k}^{-}\right)\right]
$$


in the strong operator topology. Let $x \in X$ and $\varepsilon>0$ be given. Let $\left\{\lambda_{k}\right\}_{0}^{n}$ be a partition of $J$ containing the points $\nu_{1}, \ldots, \nu_{m}$ for some $m$, such that

$$
\begin{gathered}
\left\|g(A) x-\sum_{1}^{n} g\left(\lambda_{k}\right)\left[E\left(\lambda_{k}\right)-E\left(\lambda_{k-1}\right)\right] x\right\|<\varepsilon, \\
\left\|h(A) x-\sum_{1}^{m} \rho_{k}\left[E\left(\nu_{k}\right)-E\left(\nu_{k}^{-}\right)\right] x\right\|<\varepsilon .
\end{gathered}
$$

Let $\left\{\mu_{j}\right\}_{0}^{q}$ denote the partition of $H$ consisting of all points $f\left(\lambda_{k}\right)$ where $\lambda_{k}$ is in the above partition, and include also those points $\gamma_{i}$ corresponding to $f\left(\nu_{i}\right)$. With respect to this partition,

$$
\begin{aligned}
\sum_{1}^{q} \mu_{j}\left[F\left(\mu_{j}\right)\right. & \left.-F\left(\mu_{j-1}\right)\right] \\
= & \sum_{1}^{m} f\left(\nu_{k}\right)\left[E\left(\nu_{k}\right)-E\left(\nu_{k}^{-}\right)\right]+\sum g\left(\lambda_{k}\right)\left[E\left(\lambda_{k}\right)-E\left(\lambda_{k-1}\right)\right] \\
= & \sum_{1}^{m} \rho_{k}\left[E\left(\nu_{k}\right)-E\left(\nu_{j}^{-}\right)\right]+\sum_{1}^{q} g\left(\lambda_{k}\right)\left[E\left(\lambda_{k}\right)-E\left(\lambda_{k-1}\right)\right] .
\end{aligned}
$$

Refining the $\left\{\mu_{j}\right\}$ partition, if necessary, we have

$$
\left\|B x-\sum \mu_{i}\left[F\left(\mu_{j}\right)-F\left(\mu_{j-1}\right)\right] x\right\|<\varepsilon .
$$

Since this only induces a further refinement of the partition of $J$, we have $\| B x-$ $f(A) x \|<2 \varepsilon$.

2.10. LeMmA. If $f: J \rightarrow \mathbf{R}$ is bounded and monotone nonincreasing, then $f(A)$ is well bounded of type (B).

Proof. It suffices to show that $-A$ is well bounded of type (B) if $A$ is. Directly from the definition of total variation, we see that $\|p(-A)\| \leqslant K\|p\|_{-J}$, so $-A$ is well bounded. To show that $-A$ is of type (B), it suffices to show that for every $x$ in $X$, $f \rightarrow f(-A) x$ is a compact linear map of $\mathrm{AC}(J)$ into $X$ [2, Theorem 17.14, (ii)]. Since this property holds for $A$, and $-A=g(A)$, with $g(\lambda)=-\lambda$, the result follows.

3. The case that $f$ is piecewise monotone. Let $E(\lambda)$ be a spectral family on $J$, and assume $J=\bigcup_{1}^{m} J_{i}$, wshere $J_{i}=\left[a_{i-1}, a_{i}\right], a_{0}=a, a_{m}=b$. We define subspaces $X_{i}$ of $X, i=0, \ldots, m$ :

$$
\begin{aligned}
X_{0} & =\{E(a) x: x \in X\}, \\
X_{i} & =\left\{\left[E(\lambda)-E\left(a_{i-1}\right)\right] x: x \in X, \lambda \in J_{i}\right\}, \quad i=1, \ldots, m .
\end{aligned}
$$

Using the defining properties of a spectral family, it is easy to see that each $X_{i}$ is closed, and any two have only the zero of $X$ in common. Since any $x \in X$ has the decomposition

$$
x=\sum_{i=0}^{m} x_{i}=E(a) x+\sum_{i=1}^{m}\left[E\left(a_{i}\right)-E\left(a_{i-1}\right)\right] x,
$$

we have

$$
X=X_{0} \oplus X_{1} \oplus \cdots \oplus X_{m} .
$$


In each $X_{i}$ there is a spectral family $\left\{E_{i}(\lambda): \lambda \in J_{i}\right\}$ given by

$$
\begin{gathered}
E_{0}(\lambda)=E(a), \quad \lambda \in J_{0}=\{a\}, \\
E_{i}(\lambda)=E(\lambda)-E\left(a_{i-1}\right), \quad \lambda \in J_{i} .
\end{gathered}
$$

Let

$$
A=\int_{J}^{\oplus} \lambda d E(\lambda), \quad A_{i}=\int_{J_{i}}^{\oplus} \lambda d E_{i}(\lambda)
$$

By considering a partition of $J$ including the endpoints $a_{i}$, we have

$$
A=A_{0} \oplus \cdots \oplus A_{m},
$$

and more generally, if $f \in \mathrm{BV}(J), f_{i}=\left.f\right|_{J_{i}}$, we have

$$
f(A)=f_{0}\left(A_{0}\right) \oplus \cdots \oplus f_{m}\left(A_{m}\right) .
$$

If $f$ is monotone on each $J_{i}$, then by the considerations of $\S 2$, each $B_{i}=f_{i}\left(A_{i}\right)$ is well bounded of type (B). To show that $B=f(A)$ is well bounded, let $p$ be a polynomial on $J$. Then

$$
\begin{aligned}
\|p(B)\| & \leqslant\left\|p_{0}\left(B_{0}\right)\right\|+\cdots+\left\|p_{n}\left(B_{m}\right)\right\| \\
& \leqslant K_{0}\left\|p_{0}\right\|_{J_{0}}+\cdots+K_{m}\left\|p_{m}\right\|_{J_{m}} \\
& \leqslant K\left[|p(a)|+\sum_{1}^{m} \max \left(|p|, J_{i}\right)+\sum_{1}^{m} \operatorname{var}\left(p, J_{i}\right)\right] \\
& \leqslant K\|p\|_{J} .
\end{aligned}
$$

To show that $B$ is of type (B), we again consider the compactness of the mapping of $\mathrm{AC}(J) \rightarrow X$ given by $f \rightarrow f(B) x, x$ fixed. Since the map $f_{i} \rightarrow f_{i}\left(B_{i}\right) x_{i}$ is compact, we easily see that the same holds for $B$.

The spectral family $\{F(\mu): \mu \in H\}$ of $f(A)$ can be expressed in terms of $E(\lambda)$. For given $\mu \in H$, the level set $S(\mu)$ now consists of the union of finitely many disjoint intervals, which may or may not contain some of their endpoints. Consider the following list giving a correspondence between intervals and differences of projections:

$$
\begin{array}{ll}
(\alpha, \beta), & E\left(\beta^{-}\right)-E(\alpha), \\
(\alpha, \beta], & E(\beta)-E(\alpha), \\
{[\alpha, \beta)} & E\left(\beta^{-}\right)-E\left(\alpha^{-}\right), \\
{[\alpha, \beta],} & E(\beta)-E\left(\alpha^{-}\right) .
\end{array}
$$

Then $F(\mu)$ is the sum over each interval in $S(\mu)$, of the corresponding differences of projections.

We now indicate the procedure for replacing $J$ by a simple, nonclosed rectifiable arc $C$. Let $\rho_{c}$ denote the arc-length parameterizations of $C$. Thus there is a real interval $J_{c}$ whose length is the arc-length of $C$, such that $\rho_{c}: J_{c} \rightarrow C, \rho_{c} \in \mathrm{AC}\left(J_{c}\right)$, and $\left|\rho_{c}^{\prime}\right|=1$ a.e. [3, p. 634]. Let $f \in \operatorname{BV}(C)$, and assume $f(C)$ is contained in a simple, nonclosed rectifiable arc $\Gamma$, with arc-length parameterization $\rho_{\Gamma}: J_{\Gamma} \rightarrow \Gamma$. Define $g: J_{c} \rightarrow J_{\Gamma}$ by

$$
g=\rho_{\Gamma}^{-1} \circ f \circ \rho_{c} .
$$

3.8. Definition. $f$ is piecewise monotone on $C$ if $g$ is piecewise monotone on $J_{c}$. 
3.9. TheOREM. If $A$ is well bounded of type (B) on $C$, and if $f \in \mathrm{BV}(C)$ maps $C$ into $\Gamma$, such that $f$ is piecewise monotone on $C$, then $f(A)$ is well bounded of type $(\mathrm{B})$ on $\Gamma$.

4. An application. Let $L$ be a closed operator with domain $D(L)$ dense in $X$. Assume zero is in the resolvent set of $L$, and let $R=(-L)^{-1}$.

4.1. TheOrem. Assume $R$ is a well-bounded operator of type (B) on a simple, nonclosed rectifiable arc $C$. Let $p$ be a polynomial such that

(i) $p(C)$ is contained in a simple, nonclosed rectifiable arc $\Gamma$;

(ii) $p\left(-\lambda^{-1}\right) \neq 0$ for $\lambda$ in $C-\{0\}$.

Then $p(L)$ has a well-bounded inverse of type $(\mathrm{B})$.

Proof. Let $R=\int_{C}^{\oplus} \lambda d E(\lambda)$. If $f \in \mathrm{BV}(C)$ and $\alpha>0$, let

$$
f_{\alpha}(\lambda)= \begin{cases}f(\lambda), & \rho_{c}(\lambda, 0) \geqslant \alpha, \\ 0, & \rho_{c}(\lambda, 0)<\alpha .\end{cases}
$$

Then [1, Corollary 5.13]

$$
L x=\lim _{\alpha \downarrow 0} \int_{C}^{\oplus}\left(\frac{-1}{\lambda}\right)_{\alpha} d E(\lambda) x, \quad x \in D(L),
$$

and for any polynomial $p$,

$$
p(L) x=\lim _{\alpha \downarrow 0} \int_{C}^{\oplus} p_{\alpha}\left(\frac{-1}{\lambda}\right) d E(\lambda) x, \quad x \in D(p(L)) .
$$

Let $q(\lambda)=\lambda^{n} p(-1 / \lambda)$, where $n$ is the degree of $p$. Then $q$ is also a polynomial of degree $n, q(\lambda) \neq 0$ for $\lambda$ in $C-\{0\}$, and $-p^{-1}\left(-\lambda^{-1}\right)=-\lambda^{n} q^{-1}(\lambda)$. By (ii), $-\lambda^{n} q^{-1}(\lambda)$ $\in \operatorname{BV}(C)$, so

$$
S=\int_{C}^{\oplus}-\lambda^{n} q^{-1}(\lambda) d E(\lambda)
$$

is a bounded linear operator. Using the functional calculus,

$$
-p(L) S x=\lim _{\alpha \downarrow 0} \int_{C}^{\oplus}(1)_{\alpha} d E(\lambda) x=x, \quad x \in X,
$$

and

$$
-S p(L) x=x, \quad x \in D(p(L)) .
$$

Thus $S$ is the inverse of $-p(L)$, and $S$ is well bounded of type (B) since the rational function $-\lambda^{n} q^{-1}(\lambda)$ is piecewise monotone.

\section{REFERENCES}

1. H. E. Benzinger, E. Berkson and T. A. Gillespie, Spectral families of projections, semigroups and differential operators, Trans. Amer. Math. Soc. 275 (1983), 431-475.

2. H. R. Dowson, Spectral theory of linear operators, London Math. Soc. Monographs, No. 12, Academic Press, New York, 1978.

3. J. R. Ringrose, On well-bounded operators. II, Proc. London Math. Soc. (3) 13 (1963), 613-638.

Department of Mathematics, University of Illinois, Urbana, Illinois 61801 